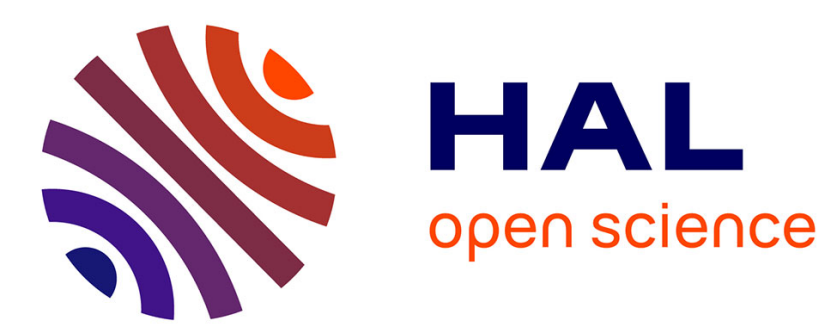

\title{
Impulsive control of the bilinear Schrödinger equation: propagators and attainable sets
}

\author{
Nabile Boussaid, Marco Caponigro, Thomas Chambrion
}

\section{To cite this version:}

Nabile Boussaid, Marco Caponigro, Thomas Chambrion. Impulsive control of the bilinear Schrödinger equation: propagators and attainable sets. 2019 IEEE 58th Conference on Decision and Control (CDC), Dec 2019, Nice, France. 10.1109/CDC40024.2019.9029277 . hal-02747636

\section{HAL Id: hal-02747636 \\ https://hal.science/hal-02747636}

Submitted on 3 Jun 2020

HAL is a multi-disciplinary open access archive for the deposit and dissemination of scientific research documents, whether they are published or not. The documents may come from teaching and research institutions in France or abroad, or from public or private research centers.
L'archive ouverte pluridisciplinaire HAL, est destinée au dépôt et à la diffusion de documents scientifiques de niveau recherche, publiés ou non, émanant des établissements d'enseignement et de recherche français ou étrangers, des laboratoires publics ou privés. 


\title{
Impulsive control of the bilinear Schrödinger equation: propagators and attainable sets
}

\author{
Nabile Boussaïd \\ Laboratoire de Mathématiques de Besançon, UMR 6623 \\ Université de Franche Comté, Besançon, France \\ Nabile.Boussaid@univ-fcomte.fr \\ Marco Caponigro \\ Équipe M2N \\ Conservatoire National des Arts et Métiers, Paris, France \\ Marco.Caponigro@cnam.fr
}

Thomas Chambrion

Université de Lorraine, IECL, UMR 7502, Vandoeuvre-lès-Nancy, F-54506, France

CNRS, IECL, UMR 7502, Vandoeuvre-lès-Nancy, F-54506, France

Inria, Villers-lès-Nancy, F-54600, France

Thomas.Chambrion@univ-lorraine.fr

\begin{abstract}
We consider a linear Schrödinger equation with an unbounded bilinear control term. The control term is the derivative of function with bounded variations (impulsive control). Well-posedness results and regularity of the associated propagators follow from classical theory from Kato. As a byproduct, one obtains a topological obstruction to exact controllability of the system in the spirit of the results of Ball, Marsden and Slemrod.
\end{abstract}

\section{INTRODUCTION}

\subsection{Bilinear Schrödinger equation}

The state of a quantum system evolving on a Riemmanian manifold $\Omega$ can be represented, in first approximation at a certain time $t$, by its wave function, $\psi(t)$ a point of $L^{2}(\Omega, \mathbf{C})$. When excited by an electric field (for instance a laser), the time evolution of $\psi$ is given by the Schrödinger equation with a bilinear control term

$$
\mathrm{i} \frac{\partial \psi}{\partial t}(x, t)=(-\Delta+V(x)) \psi(x, t)+u(t) W(x) \psi(x, t)
$$

where $\Delta$ is the Laplace-Beltrami operator associated with the metric of $\Omega, V: \Omega \rightarrow \mathbf{R}$ is a function accounting for the physical properties of $\Omega$ (especially: confining properties) in the absence of excitation, $W: \Omega \rightarrow \mathbf{R}$ represents the physical properties of the laser and $u: \mathbf{R} \rightarrow \mathbf{R}$ is the control. An initial condition $\psi(0)=\psi_{0}$ is assigned once for all. 
The aim of the quantum control is to chose a suitable $u$ in such a way that the solution $\psi$ of (1) (associated with the initial condition) enjoys some desirable properties, for instance, converges to a given target.

Under physically reasonable assumptions on the potentials $V$ and $W$, the linear operator i $(\Delta-$ $V+u W$ ) generates a $C^{0}$ semi group of unitary operators $t \mapsto \Upsilon^{u}$ for every $u$ in $\mathbf{R}$. Consequently, one defines the solution of (1) for piecewise constant control $u:[0,+\infty) \rightarrow \mathbf{R}$ by concatenation of propagators for constant $u$ by

$$
\Upsilon_{t, 0}^{u} \psi_{0}=e^{\left(t-t_{n}\right)\left(A+u_{n} B\right)} \circ \cdots \circ e^{t_{1}\left(A+u_{1}\right) B} \psi_{0}
$$

if $u=\sum_{j \geq 0} u_{j} \mathbf{1}_{\left[t_{j}, t_{j+1}\right)}$ and $t \in\left(t_{n}, t_{n+1}\right)$.

This problem of extension of $\Upsilon$ to more general controls (not only piecewise constant) is motivated by recent technical developments in quantum sciences. It is now technically possible to induce very short laser pulses ([ABGM05], [CM05]) corresponding to a control $u$ with a very small support in (1). A natural question is to give a meaning and to prove the existence of solutions to (1) for very irregular controls $u$, including sums of Dirac masses. In this case, $u$ is a measure and not a function anymore.

\subsection{Impulsive control}

The problem of considering measures instead of functions in control problems is not new. A vast literature of the last century considers finite dimensional systems of the form

$$
y^{\prime}=f(y, t)+\sum_{j=1}^{n} u_{j}(t) g_{j}(y, t)
$$

where $f, g_{1}, \ldots, g_{n}$ are regular vector fields on $\mathbf{R}^{d}$ and $u_{j}$ are the derivatives of functions with bounded variations (i.e., Radon measures). After considerable efforts, the situation for finite dimensional dynamics of the type of (2) (and for others) is now well understood. Let us cite, among many other contributions, [Sus76], [Mil76], [DMR91], [BR88], [Bre96], [Bre08].

Much less work has been done in the infinite dimensional framework, mostly when the control has a discrete set of atoms [BKMZ94], or involving continuous control operators [IL18].

\subsection{Main results}

Let $T>0, u$ a Radon measure on $[0, T]$ (basic definitions and properties of Radon measures are recalled in the Appendix), $v$ a primitive of $u$ with bounded variation such that $v(0)=0$, and $\psi_{0}$ in $L^{2}(\Omega, \mathbf{C})$.

Definition 1. We say that $\psi:[0, T] \rightarrow D(W)$ is a classical mild solution of (1) with initial condition $\psi_{0}$ associated with $u$ if for every $t$ in $[0, T]$,

$$
\begin{aligned}
\psi(x, t) & =e^{-\mathrm{i} t(-\Delta+V(x))} \psi_{0}(x) \\
& -\int_{s=0}^{t} u(s) e^{-\mathrm{i}(t-s)(-\Delta+V(x))} \mathrm{i} W(x) \psi(s, x) \mathrm{d} s .
\end{aligned}
$$

Definition 2. We say that $\psi:[0, T] \rightarrow D(-\Delta+V)$ is a generalized impulsive mild solution of (1) with initial condition $\psi_{0}$ associated with $v$ if for every $t$ in $[0, T]$,

$$
\begin{aligned}
& \psi(x, t)=e^{-\mathrm{i} v(t) W(x)} \psi_{0}(x) \\
& \quad-\int_{s=0}^{t} e^{-\mathrm{i}(v(t)-v(s)) W(x)} \mathrm{i}(-\Delta+V(x)) \psi(s, x) \mathrm{d} s .
\end{aligned}
$$


We focus on a class of bilinear equations that are perturbations of the controlled quantum harmonic oscillator. We consider $\Omega=\mathbf{R}, V: x \in \mathbf{R} \mapsto x^{2}+V_{1}(x), W: x \in \mathbf{R} \mapsto x+V_{2}(x)$ for $V_{1}$ and $V_{2}$ arbitrary smooth (i.e. $C^{\infty}$ ) functions with compact support. Equation (1) then reads

$$
\mathrm{i} \frac{\partial \psi}{\partial t}(x, t)=\left(-\Delta+x^{2}+V_{1}(x)+u(t)\left(x+V_{2}(x)\right)\right) \psi(x, t) .
$$

We denote by $\mathcal{H}^{2 k}=D\left(\left|-\Delta+x^{2}\right|^{k}\right)$, being the pertubation $V_{1}$ bounded we have that $\mathcal{H}^{2 k}=$ $D\left(\left|-\Delta+x^{2}+V_{1}(x)\right|^{k}\right)$. Our first result is an existence and uniqueness result for (5) in presence of impulsive controls.

Theorem 1. Given $k>0$ and $\psi_{0}$ in $\mathcal{H}^{2 k}$ there exists a unique generalized impulsive mild solution $t \mapsto \Upsilon_{t}^{\mathrm{d} v} \psi_{0}$ of (5) with initial condition $\psi_{0}$ associated with $v$. More generally, if a sequence $\left(v_{n}\right)_{n \in \mathbf{N}}$ of functions $v_{n}:[0, T] \rightarrow \mathbf{R}$ has uniformly bounded total variation and converges pointwise to $v:[0, T] \rightarrow$ $\mathbf{R}$ with bounded variation, then for every $\psi_{0}$ in $\mathcal{H}^{2 k}$, the sequence $\left(\Upsilon_{T}^{\mathrm{d} v_{n}} \psi_{0}\right)_{n \in \mathbf{N}}$ converges to $\Upsilon_{T}^{\mathrm{d} v} \psi_{0}$ in the graph norm of $\mathcal{H}^{2 k}$.

Proposition 2. Let $k>0$ and $\psi_{0}$ be in $\mathcal{H}^{2 k}$. If $v$ is a absolutely continuous with respect to Lebesgue measure whose density has bounded variations (i.e., its derivative $u=v^{\prime}$ has bounded variations), then the generalized impulsive mild solution $\Upsilon_{T}^{\partial v} \psi_{0}$ of (5) is a classical mild solution.

Theorem 3. For every $k>0$, for every $\psi_{0}$ in $\mathcal{H}^{2 k}$, the attainable set of (5) from $\psi_{0}$ with Radon measures

$$
\bigcup_{T \geq 0} \bigcup_{u \in \mathcal{R}([0, T])} \bigcup_{\substack{v \in B V \\ v^{\prime}=u}}\left\{\Upsilon_{t}^{\partial v} \psi_{0}, 0 \leq t \leq T\right\}
$$

is meager in $\mathcal{H}^{2 k}$ and, hence as an empty interior in the unit sphere of $L^{2}(\mathbf{R}, \mathbf{C})$.

A consequence of this result is that the perturbed quantum harmonic oscillator (5) is non-controllable. The fact that the quantum harmonic oscillator $\left((5)\right.$ with $\left.V_{1}=0=V_{2}\right)$ is not controllable in any reasonable sense by means of piecewise constant controls is a well-known result, see [MR04]. Heuristically, the system enjoys too many symmetries to be controllable. Here we show that the non-controllability holds even when enlarging the class of controls. Moreover, Theorem 3 states that every (smooth) perturbation of the quantum harmonic oscillator is not exactly controllable. This result is in the spirit of [BMS82], see also [BCC19] for a general discussion on attainable sets associated with $L^{1}$ controls. However, notice that generically system (5) is approximately controllable by means of piecewise constant controls [MS10].

\subsection{Originality of the contribution}

Theorem 1 is mainly based on results of Section 5 in [BCC17]. It is a generalization of a result of Fujiwara ([Fuj79] and [Car11]) dealing with locally bounded control $u$ (i.e., $v$ is Lipschitz continuous). The paper [BCC17] is technically involved and addresses the case of systems way more less regular than (5). The present paper aims to take advantage of the smoothness of the potentials appearing in system (5) and the abstract results of [BCC17] to give a simpler (and much shorter) presentation of the rather simple underlying ideas. 


\subsection{Content}

Some notations used troughout the paper are introduced in Section 2. The main difficulty when dealing with measures as controls is the possible presence of atoms, as explained in Section 3 . In Section 4 we give the definition of propagators and we present the first properties used in the proofs of Theorem 1 and Proposition 2 given in Section 5. The proof of Proposition 3 is presented in Section 6.

\section{PRELIMINARIES}

\subsection{Abstract framework}

We rewrite equation (1) in a more abstract form as

$$
\psi^{\prime}=A \psi+u(t) B \psi
$$

where $A=-\mathrm{i}(\Delta+V)$ and $B=-\mathrm{i} W$ are linear operators on a Hilbert space $\mathcal{H}\left(L^{2}(\mathbf{R}, \mathbf{C})\right.$ for Theorem 1) endowed with its Hilbert product $\langle\cdot, \cdot\rangle$ (the standard $L^{2}$ Hilbert product for the case of Theorem 1). This operator framework is used for the analysis in Sections 3 and 4 below.

\subsection{Notations}

In what follows, let $I$ be an interval $I \subset \mathbf{R}$ containing zero. Let $P C(I, K)$ be the set of piecewise constant functions from $I$ taking value in $K$ and $\mathcal{R}(I)$ be the set of Radon measures on $I$. For the reader's convenience, basic facts about Radon measures are presented in Appendix.

\section{CONTINUITY WITH RESPECT TO MEASURES}

The rationale for the introduction of the notion of generalized impulsive mild solution is topological. Indeed consider, for instance, two sequences of Radon measures $\left(\mu_{n}\right)_{n \in \mathbf{N}}$ and $\left(\nu_{n}\right)_{n \in \mathbf{N}}$, defined for $n$ in $\mathbf{N}$ by

$$
\mu_{n}=\delta_{1-\frac{1}{n}} \quad \text { and } \quad \nu_{n}=\delta_{1+\frac{1}{n}} .
$$

The two sequences $\left(\mu_{n}\right)_{n \in \mathbf{N}}$ and $\left(\nu_{n}\right)_{n \in \mathbf{N}}$ converge toward $\delta_{1}$ for the weak and the narrow topologies, none of them converges to anything for the total variation topology (see Subsection A.4 of Appendix for definitions). In this section, we use these sequences to present the intuition behind the definition of solutions.

\subsection{Shock modeling}

We consider dynamics of the type (6) where $A$ and $B$ are linear operators with domain $D(A) \subset \mathcal{H}$ and $D(B) \subset \mathcal{H}$, respectively, such that for every $u$ in $\mathbf{R}, A+u B$ is defined on a dense subset $D(A+u B)$ of $\mathcal{H}$ and generates a $C^{0}$ semi-group of linear operators. Under these assumption one can define the propagator $u \mapsto \Upsilon_{T}^{u}$ of (6) associated with a piecewise constant control $u=\sum_{j \geq 0} u_{j} \mathbf{1}_{\left[t_{j}, t_{j+1}\right)}$ as the concatenation of propagators

$$
\Upsilon_{t}^{u} \psi_{0}=e^{\left(t-t_{n}\right)\left(A+u_{n} B\right)} \circ \cdots \circ e^{t_{1}\left(A+u_{1}\right) B}
$$

for $t \in\left(t_{n}, t_{n+1}\right)$. 
Proposition 4. Let $T$ be a positive real, in general, the mapping $\Upsilon_{T}: P C([0, T], \mathbf{R}) \rightarrow L(\mathcal{H}, \mathcal{H})$ does not admit an extension to $\mathcal{R}([0, T])$ continuous for the weak topology.

Proof. Let us consider the case in which $\mathcal{H}$ is finite dimensional. The extension to the general infinite dimensional framework follows from Hahn-Banach extension theorem. In the finite dimensional case, any linear operator $A: D(A) \rightarrow$ defined on any dense subset $D(A)$ of $\mathcal{H}$ has a unique continuous extension to $\mathcal{H}$. It is also the generator of a $C^{0}$ semi-group of linear operators and the exponential $e^{t A}$ is defined as the convergent sum $e^{t A}=\sum_{k=0}^{\infty} \frac{A^{k}}{k !}$.

For every $n$ in $\mathbf{N}$, define $u_{n}:[0,2] \rightarrow \mathbf{R}$ by $u_{n}=n \mathbf{1}_{\left(1-\frac{1}{n}, 1\right)}$, that is $u_{n}(x)=n$ if and only if $x \in\left(1-\frac{1}{n}, 1\right)$, and $u_{n}(x)=0$ otherwise. Similarly, for every $n$ in $\mathbf{N}$, define $v_{n}:[0,2] \rightarrow \mathbf{R}$ by $v_{n}=n \mathbf{1}_{\left(1,1+\frac{1}{n}\right)}$, that is $v_{n}(x)=n$ if and only if $x \in\left(1,1+\frac{1}{n}\right)$, and $v_{n}(x)=0$ otherwise, and $w_{n}=\frac{u_{n}+v_{n}}{2}$.

The sequences $\left(u_{n}\right)_{n \in \mathbf{N}},\left(v_{n}\right)_{n \in \mathbf{N}}$ and $\left(w_{n}\right)_{n \in \mathbf{N}}$, seen as sequences of measures, converge to $\delta_{1}$ in the weak sense but do not converge in the strong sense.

One computes, for every $n$ in $\mathbf{N}$,

$$
\begin{aligned}
\Upsilon_{1}^{u_{n}} & =e^{B} \circ e^{A} \\
\Upsilon_{1}^{v_{n}} & =e^{A} \\
\Upsilon_{1}^{w_{n}} & =e^{\frac{1}{2} B} \circ e^{A} .
\end{aligned}
$$

The result follows by noting that these limits do not coincide when one chooses $B$ such that $e^{B} \neq$ $\operatorname{Id}_{\mathcal{H}}$.

Proposition 5. Let $\mathcal{H}$ be finite dimensional, $\psi_{0} \neq 0$ in $\mathcal{H}, A, B: \mathcal{H} \rightarrow \mathcal{H}$ be two linear operators such that $B^{2} e^{A} \psi_{0} \neq 0$. Define $u_{n}=n \mathbf{1}_{(1-1 / n, 1)}$ and $\psi_{n}$ by

$$
\begin{aligned}
t & \mapsto e^{t A} \psi_{0} \text { if } 0 \leq t<1-1 / n \\
t & \mapsto e^{\left(t-1+\frac{1}{n}\right)(A+n B)} e^{\left(1-\frac{1}{n}\right) A} \psi_{0} \text { if } 1-\frac{1}{n} \leq t<1 \\
t & \mapsto e^{(t-1) A} e^{A / n+B} e^{\left(1-\frac{1}{n}\right) A} \psi_{0} \text { if } t \geq 1
\end{aligned}
$$

Then (i) $\psi_{n}$ is a mild solution of (6) associated with control $u_{n}$, (ii) $u_{n}$ converges weakly (as a Radon measure) to $\delta_{1}$, (iii) the sequence $\psi_{n}$ pointwise converges to a $B V$ function $\psi$ but (iv) $\psi$ is not a classical mild solution of (6) associated with control $\delta_{1}$.

Even when the ambient space $\mathcal{H}$ is one dimensional, it is not easy to give a meaning to (3) when $u=\delta_{0}+\delta_{1}$ and $t=0$ or $t=1$ : should the bounds 0 and 1 of the integral be included or not?

The common reason underlying this question, as well as Propositions 4 and 5 , is the presence of atoms ("Dirac masses") in the limit control $u$. Indeed, the question is always to know whether the shock (modeled by an atom of $u$ at $t$ ) takes place before or after the time $t$. Recall that, despite the name "discret part", such atoms may accumulate. For instance, the set of atoms of Radon measure may be dense in $\mathbf{R}$. This prevents a point by point treatment of the jumps.

Quite surprisingly, such phenomena do not occur with singular continuous controls without discrete part. 


\section{$3.2 \quad$ A topological choice}

The generalized notion of solution (Definition 2) associates a solution of (1) with a primitive of the control, not with the control itself. The value chosen for the primitive at atoms of the control is arbitrary and accounts for the time when the shock occurs. For shocks happening just before the atom, the chosen primitive will be left continuous. For shocks beginning just after the atom, the chosen primitive will be right continuous. Other choices of the value of the primitive will account for more complex situations.

\section{MILD COUPLING APPROACH}

\subsection{Heuristic}

The basic idea in our approach is to introduce a variable $z(t)=e^{-v(t) B} \psi(t)$ where $v$ is a primitive of the control $u$ vanishing at zero, for instance the left continuous one $v(t)=u([0, t))$. The new unknown $z$ satisfies the differential equation:

$$
z^{\prime}(t)=e^{-v(t) B} A e^{v(t) B} z(t)
$$

with initial condition $z(0)=\psi_{0}$. Then

$$
z(t)=z(0)+\int_{0}^{t} e^{-v(s) B} A e^{v(s) B} z(s) \mathrm{d} s
$$

which provides a generalized impulsive mild solution after the change of variable $\psi(t)=e^{v(t) B} z(t)$. At this stage, all these derivations are purely formal. The aim of the following is to provide a rigorous construction of this heuristic.

\subsection{Classical propagator}

Since the problem (1) is nonautonomous, the notion of semi-group is replaced by the following

Definition 3 (Propagator on a Hilbert space). A family $(s, t) \in \Delta_{I} \mapsto X(s, t)$ of linear contractions on a Hilbert space $\mathcal{H}$, strongly continuous in $t$ and $s$ and such that

(i) $X(t, s)=X(t, r) X(r, s)$, for any $s<r<t$,

(ii) $X(t, t)=\operatorname{Id}_{\mathcal{H}}$,

is called a contraction propagator on $\mathcal{H}$.

Following [Kat53] in the construction of propagators, we introduce the following

Assumption 1. Let $\mathcal{D}$ be a dense subset of $\mathcal{H}$

1. $A(t)$ is a maximal dissipative operator on $\mathcal{H}$ with domain $\mathcal{D}$ for every $t \in I$,

2. $t \mapsto A(t)$ has bounded variation from $I$ to $L(\mathcal{D}, \mathcal{H})$, where $\mathcal{D}$ is endowed with the graph topology associated with $A(a)$ for some $a \in I$,

3. $M:=\sup _{t \in I}\left\|(1-A(t))^{-1}\right\|_{L(\mathcal{H}, \mathcal{D})}<\infty$. 
Theorem 6. If $t \in I \mapsto A(t)$ satisfies Assumption 1, then there exists a unique contraction propagator $X: \Delta_{I} \rightarrow L(\mathcal{H})$ such that if $\psi_{0} \in \mathcal{D}$ then $X(t, s) \psi_{0} \in \mathcal{D}$ and is strongly right differentiable in $t$ with derivative $A(t+0) X(t, s) \psi_{0}$.

Moreover, with $M$ from Assumption 3,

$$
\left\|A(t) X(t, s) \psi_{0}\right\| \leq M e^{M \operatorname{TV}(A,(I, L(\mathcal{D}, \mathcal{H})))}\left\|A(s) \psi_{0}\right\|
$$

for $(t, s) \in \Delta_{I}$ and $\psi_{0} \in \mathcal{D}$, and, moreover, $X(t, s) \psi_{0}$ is left differentiable in $s$ with derivative $-A(s-$ 0) $\psi_{0}$ when $t=s$.

In the case in which $t \mapsto A(t)$ is continuous and skew-adjoint, if $\psi_{0} \in \mathcal{D}$ then $t \in(s,+\infty) \mapsto$ $X(t, s) \psi_{0}$ is strongly continuously differentiable in $\mathcal{H}$ with derivative $A(t) X(t, s) \psi_{0}$.

\subsection{Generalized propagator}

Assumption 2. $(A, B)$ is a pair such that

1. A is a maximal dissipative operator on $\mathcal{H}$ with domain $D(A)$,

2. there exist $c \geq 0$ and $c^{\prime} \geq 0$ such that $B-c$ and $-B-c^{\prime}$ generate contraction semi-groups on $\mathcal{H}$ leaving $D(A)$ invariant,

3. the map $t \in \mathbf{R} \mapsto e^{t B} A e^{-t B} \in L(D(A), \mathcal{H})$ is locally Lipschitz.

Applying Theorem 6 in the framework of Assumption 2 with $A(t):=e^{-v(t) B} A e^{v(t) B}$, one gets the existence of generalized impulsive mild solution.

Definition 4. Let $(A, B)$ satisfy Assumption 2. Let $u \in \mathcal{R}[0, T]$. For any $v \in B V([0, T])$ with distributional derivative $u$ let $t \mapsto Y_{t}^{u}$ be the contraction propagator with initial time $s=0$ associated with $A(t):=e^{-v(t) B} A e^{v(t) B}$. We define a generalized propagator associated with $A+u(t) B$ with initial time zero, to be $\Upsilon_{t}^{\mathrm{d} v}=e^{v(t) B} Y_{t}^{u}$ for every $t$ in $[0, T]$ and $v$ in $B V([0, T])$ such that $v^{\prime}=u$ in the distributional sense.

Proposition 7. With the notations of Definition 4, for every $\psi_{0} \in D(A), \Upsilon_{t}^{\mathrm{d} v} \psi_{0}$ is the unique generalized impulsive mild solution at time $t$ of (6) associated with $v$.

Notice that a Radon measure admits an infinity of primitives with bounded variations vanishing at zero if and only if this measure has atoms. In other words, the generalized propagator defined in Definition 4 is uniquely defined at every time where $u$ has no atoms. On the atoms of $u$, the value of the primitive $v$ is arbitrary.

The result of Proposition 7 can be rewritten in spaces of the form $D\left(A^{k}\right)$ instead of $\mathcal{H}$ as soon as $B$ preserves the the domains of $A$ in the following sense:

Definition 5. Let $k$ be a nonnegative real. A pair of skew-adjoint operators $(A, B)$ is $k$-mildly coupled if

(i) $A$ is invertible with bounded inverse from $D(A)$ to $\mathcal{H}$,

(ii) for any real $t, e^{t B} D\left(|A|^{k / 2}\right) \subset D\left(|A|^{k / 2}\right)$,

(iii) there exists $c \geq 0$ and $c^{\prime} \geq 0$ such that $B-c$ and $-B-c^{\prime}$ generate contraction semi-groups on $D\left(|A|^{k / 2}\right)$ for the norm $\|\cdot\|_{k / 2}:=\left\||A|^{k / 2} \cdot\right\|$. 
Under the mild-coupling assumption, Proposition 7 can be extended to higher regularity spaces, as in Propositions 8 and 9 below.

Proposition 8. Let $k$ be a positive real. Let $(A, B)$ satisfy Assumption 2 and be $k$-mildly coupled. Then there exists $C>0$ such that for every $s \in[0, k], \psi_{0} \in D\left(|A|^{s / 2}\right)$, for every $T \geq 0$, one has $\Upsilon_{T}^{\mathrm{o} v}\left(\psi_{0}\right) \in D\left(|A|^{s / 2}\right)$ and

$$
\left\|\Upsilon_{T}^{\mathrm{\partial} v}\left(\psi_{0}\right)\right\|_{s / 2} \leq e^{\frac{s}{k} C \operatorname{TV}(v,([0, T], \mathbf{R}))}\left\|\psi_{0}\right\|_{s / 2}
$$

for every $v$ in $B V([0, T], K)$ with derivative $v^{\prime}=u \in \mathcal{R}[0, T]$.

Proof. This is Proposition 25 of [BCC17].

Proposition 9. Let $T$ be a positive real, let $k$ be a positive real, let $(A, B)$ satisfy Assumption 2, and let $(A, B)$ be $k$-mildly coupled. Then for any $s \in[0, k)$, for every $\psi_{0}$ in $D\left(|A|^{s / 2}\right)$, the end-point mapping

$$
\begin{aligned}
\Upsilon\left(\psi_{0}\right): B V([0, T], \mathbf{R}) & \rightarrow D\left(|A|^{s / 2}\right) \\
v & \mapsto \Upsilon_{T}^{\text {dv }}\left(\psi_{0}\right)
\end{aligned}
$$

is continuous.

Proof. This is Proposition 26 of [BCC17].

\section{EXISTENCE OF GENERALIZED IMPULSIVE MILD SOLU- TIONS}

\subsection{Scheme of the proof of Theorem 1}

We are now ready to prove Theorem 1 and Proposition 2.

Lemma 10. With the Assumptions of Theorem 1, $(\mathrm{i}(-\Delta+V), \mathrm{i} W)$ is k-mildly coupled fro every $k>0$.

Proof. First, notice that if $V_{1}=W_{1}=0,(\mathrm{i}(-\Delta+V), \mathrm{i} W)$ is $k$-mildly coupled for every $k>0$. The first condition, item $(i)$ of Definition 5 is ensured by the well known (purely pointwise) spectrum $\left(\mathrm{i} \frac{k+1}{2}\right)_{k \geq 0}$ of the harmonic oscillator.

Assumption 2 and items ( $i i)$ and (iii) of Definition 5 are consequences of Lemma 19 in [BCC17] and Section IV.E of [BCC13].

For general smooth perturbations with compact support $V_{1}$ and $W_{1}$, first notice that for every $k>0$, the domain of $\left|-\Delta+x^{2}+V_{1}\right|^{k}$ and the domain of $\left|-\Delta+x^{2}\right|^{k}$ coincide. Then, notice that the multiplication by $W_{1}$ is bounded from $\left|-\Delta+x^{2}\right|^{k}$ to itself. Conclusion follows from Lemma 19 in $[\mathrm{BCC} 17]$.

The proof of Theorem 1 is now a simple consequence of the results of Section 4 . Since (i $(-\Delta+$ $V), \mathrm{i} W)$ is $k$-mildly coupled for every $k>0$, we use Definition 4 in $D\left(|-\Delta+V|^{\frac{k}{2}}\right)$ for every $k$ and, by Theorem $6, Y_{t}^{\text {ov }}$ from Definition 4$)$, lets $D\left(|-\Delta+V|^{\frac{3}{2}}\right)$ invariant and satisfies

$$
Y_{t}^{\mathrm{d} v} \psi_{0}=\psi_{0}+\int_{0}^{t} e^{-\mathrm{i} v(s) W}(-\Delta+V) e^{\mathrm{i} v(s) W} Y_{s}^{\mathrm{d} v} \psi_{0} \mathrm{~d} s .
$$

Theorem 1 follows by multiplication by $e^{\mathrm{i} v W}$. 


\subsection{Link with classical mild solution}

Since the multiplication by $W$ is bounded with respect to $-\Delta+V$ with bound zero, Theorem 6 applied with $A(t)=A+u(t) B$ ensures that, if $u$ has bounded variation (for instance if $u$ is piecewise constant), and the initial condition $\psi_{0}$ belongs to $D(-\Delta+V)$, then the system (5) admits a unique strong solution (which is also a classical mild solution). Since the generalized propagator introduced in Definition 3 coincides with classical mild solutions for piecewise constant controls, then the same is true by continuity for control with bounded variations, which proves Proposition 2.

\section{A TOPOLOGICAL OBSTRUCTION TO CONTROLLABILITY}

\subsection{BMS obstructions}

In the general case in which $\mathcal{X}$ is a Banach space, assume that $P C(K)$ is endowed with a topology for which $u \mapsto \Upsilon_{T}^{u} \psi_{0}$ is continuous for every $T>0$ and every $\psi_{0}$ in $\mathcal{X}$, and that $u \mapsto \Upsilon_{T}^{u} \psi_{0}$ admits a (necessarily unique) continuous extension to $\mathcal{Z} \supset P C(K)$. If $\mathcal{Z}_{0} \subset \mathcal{Z}$, endowed with a topology finer than the one induced by $\mathcal{Z}$, is sequentially compact (for its own topology), then for every $\psi_{0}$ in $\mathcal{X}$, for every $T>0$, the attainable set at time $T$ from $\psi_{0}$ with controls in $\mathcal{Z}_{0},\left\{\Upsilon_{T}^{u} \psi_{0} \mid u \in \mathcal{Z}_{0}\right\}$ is compact.

If $\left(\mathcal{Z}_{k}\right)_{k \in \mathbf{N}}$ is a countable covering of $\mathcal{Z}, \mathcal{Z}=\cup_{k \in \mathbf{N}} \mathcal{Z}_{k}, \mathcal{Z}_{k}$ is sequentially compact for every $i$, and the topology of $\mathcal{Z}_{i}$ is finer than the topology induced by $\mathcal{Z}$, then the attainable set at time $T$ from $\psi_{0}$ with controls in $\mathcal{Z},\left\{\Upsilon_{T}^{u} \psi_{0} \mid u \in \mathcal{Z}\right\}=\cup_{i \in \mathbf{N}}\left\{\Upsilon_{T}^{u} \psi_{0} \mid u \in \mathcal{Z}_{k}\right\}$ is a countable union of compact sets in $\mathcal{X}$ (hence is a meager set in the sense of Baire as soon as $\mathcal{X}$ is infinite dimensional).

Notice that if the input-output mapping $P C(K) \ni u \mapsto \Upsilon^{u} \psi_{0} \in C^{0}([0, T], \mathcal{X})$ is continuous, then the above results can be generalized to show that the attainable set from $\psi_{0}$ at time less than $T$ : $\cup_{0 \leq t \leq T}\left\{\Upsilon_{t}^{u} \psi_{0} \mid u \in \mathcal{Z}\right\}=\cup_{k \in \mathbf{N}} \cup_{0 \leq t \leq T}\left\{\Upsilon_{T}^{u} \psi_{0} \mid u \in \mathcal{Z}_{k}\right\}$ is a union of relatively compact sets.

This principle is an abstraction of the proof of of the following result by Ball, Marsden, and Slemrod.

Theorem 11 (Theorem 3.6 in [BMS82]). Let $\mathcal{X}$ be an infinite dimensional Banach space, A generate $a C^{0}$ semi-group of bounded linear operators on $\mathcal{X}$, and $B$ be a bounded linear operator on $\mathcal{X}$. Then for any $T \geq 0$, the input-output mapping $u \mapsto \Upsilon_{T}^{u}$ admits a unique continuous extension to $L^{1}([0, T], \mathbf{R})$ and the attainable set

$$
\bigcup_{r>1} \bigcup_{T \geq 0} \bigcup_{u \in L^{r}([0, T], \mathbf{R})}\left\{\Upsilon_{t}^{u} \psi_{0}, t \in[0, T]\right\}
$$

is contained in a countable union of compact subsets of $\mathcal{X}$, and, in particular, has dense complement.

In this case, for any $T \geq 0, \mathcal{Z}=\cup_{r>1} L^{r}([0, T], \mathbf{R})$ endowed with weak-* topology, $\mathcal{Z}_{k, j}=$ $\cup_{r \geq 1+\frac{1}{j}}\left\{f \in L^{r}([0, T], \mathbf{R}),\|f\|_{L^{r}([0, T])} \leq k\right\}$ and the sequential-compactness of $\mathcal{Z}_{k, j}$ is granted by Banach-Alaoglu-Bourbaki Theorem. The main difficulty in [BMS82] is to prove the continuity of the input-output mapping $u \mapsto \Upsilon^{u} \psi_{0}$ for the weak-* topology.

Remark 1. The above argument does not hold anymore if one considers controls in $L^{1}$, since $L^{1}$ is not a reflexive space. This is the content of [BMS82, Remark 3.8], where the question of possible extensions of the above result to $r=1$ is left open except in the so-called (see [BMS82]) diagonal case, see [BMS82, Theorem 5.5]. 


\subsection{Mild coupling}

From Proposition 9, the input-output mapping associated with the control systems appearing in Theorem 1 is continuous from the set of Radon measures to $\mathcal{H}^{2 k}$. From Helly's selection theorem, we deduce that, for every $j, k$ in $\mathbf{N}$, the attainable set from $\psi_{0}$ in time less than $j$, with Radon measures having a primitive with total variation less than $K$,

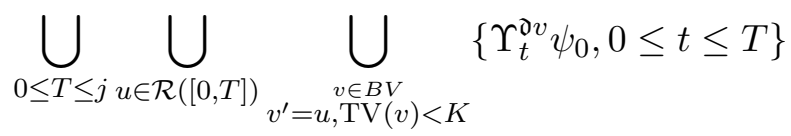

is relatively compact in $\mathcal{H}^{2 k}$, hence has empty interior by Baire's theorem.

\section{CONCLUSION}

We introduced an extension the classical notion of mild solutions to impulsive control systems with an unbounded bilinear term. Deep topological obstructions, already known for infinite dimensional bilinear control system with more regular controls in the spirit of [BMS82], still hold true with impulsive controls. As a consequence we proved a general non-controllability result for a class of quantum harmonic oscillators.

\section{A RADON MEASURES}

The aim of this appendix is to recall basic facts and definitions about Radon measures.

\section{A.1 Definitions}

Definition 6. A subset of $\mathbf{R}$ is a Borel set if it can be obtained through countable union, countable intersection and relative complements of open sets.

Definition 7. A measure on $\mathbf{R}$ is inner regular if the measure of any Borel set $B$ is the supremum of the measures of all the compact subsets of $B$.

Definition 8. A measure on $\mathbf{R}$ is outer regular if the measure of any Borel set $B$ is the infimum of the measures of all open sets containing $B$.

Definition 9. A measure on $\mathbf{R}$ is locally finite if the measure of any compact set is finite.

Definition 10. A measure on $\mathbf{R}$ is a Radon measure if it is inner regular, outer regular and locally finite.

Definition 11. A point $x$ of $\mathbf{R}$ is an atom of a Radon measure $\mu$ if $\mu(\{x\}) \neq 0$.

\section{A.2 Convergences of Radon measures}

Let $E \subset \mathcal{X}$ for $\mathcal{X}$ Banach space. A family $t \in I \mapsto u(t) \in E$ is in $B V(I, E)$, i.e. is a bounded variation function from $I$ to $E$, if there exists $N \geq 0$ such that

$$
\sum_{j=1}^{n}\left\|u\left(t_{j}\right)-u\left(t_{j-1}\right)\right\|_{\mathcal{X}} \leq N
$$


for any partition $\left(t_{j}\right)_{j=0}^{n}$ of the interval $I$. The mapping

$$
u \in B V(I, E) \mapsto \sup _{\left(t_{j}\right)_{j}} \sum_{j=1}^{n}\left\|u\left(t_{j}\right)-u\left(t_{j-1}\right)\right\|_{\mathcal{X}}
$$

is a semi-norm on $B V(I, E)$ denoted by $\mathrm{TV}(\cdot,(I, E))$ and it is called total variation.

The space $B V(I, E)$ endowed with the norm $\|\cdot\|_{B V(I)}:=\|\cdot\|_{L^{1}}+\mathrm{TV}(\cdot,(I, E))$ is a Banach space.

In what follows we consider, on $B V(I, E)$, the topology associated with the convergence given below: $\left(u_{n}\right)_{n \in \mathbf{N}} \in B V(I, E)$ converges to $u \in B V(I, E)$ if $\left(u_{n}\right)_{n \in \mathbf{N}}$ is a bounded sequence in $B V(I, E)$ pointwise convergent to $u \in B V(I, E)$.

Notice that convergence in the norm $\|\cdot\|_{B V(I)}$ implies pointwise convergence.

The Jordan Decomposition Theorem provides that any bounded variation function is the difference of two nondecreasing bounded functions. This fact, together with Helly's Theorem provides the wellknown Helly's Selection Theorem (see for example [Hel12, Nat55]).

\section{A.3 Helly's selection theorem}

Theorem 12 (Helly's selection theorem). Let I be compact and $\left(f_{n}\right)_{n \in \mathbf{N}}$ be a sequence in $B V(I, \mathbf{R})$. If

(i) there exists $M>0$ such that for all $n \in \mathbf{N}$, $\operatorname{TV}\left(f_{n},(I, \mathbf{R})\right)<M$,

(ii) there exists $x_{0} \in I$ such that $\left(f_{n}\left(x_{0}\right)\right)_{n \in \mathbf{N}}$ is bounded.

Then $\left(f_{n}\right)_{n \in \mathbf{N}}$ has a pointwise convergent subsequence.

\section{A.4 Convergence of measures}

As antederivatives of Radon measures coincide with bounded variation functions, the set of finite Radon measures on $X$, denoted by $\mathcal{R}(X)$, inherits of a total vation topology provided by the norm:

$$
\|\mu\|_{\mathcal{R}(X)}:=\sup \left\{\sum_{j \in \mathbf{N}}|\mu|\left(B_{j}\right):\left\{B_{j}\right\} \text { is a partition of } X\right\} .
$$

As any vector space, it can be endowed with its weak topology, the roughest toplogy making bounded linear functional on $\left(\mathcal{R}(X),\|\cdot\|_{\mathcal{R}(X)}\right)$ continuous. Nonetheless, we adopt here a different notion of weak convergence. We consider that $\mu_{n}$ converges to $\mu$ if and only if

$$
\int f \mathrm{~d} \mu_{n} \rightarrow \int f \mathrm{~d} \mu
$$

for every continuous function $f: X \rightarrow \mathbf{C}$ with compact support as in [EG92, Section 1.9].

If $X$ is a topological space the narrow topology is such that $\mu_{n}$ converges to $\mu$ if and only if

$$
\int f \mathrm{~d} \mu_{n} \rightarrow \int f \mathrm{~d} \mu
$$

for every bounded continuous function $f: X \rightarrow \mathbf{C}$. 


\section{ACKNOWLEDGMENTS}

This work has been partially supported by the CNRS InFiniTi DISQUO program and the ANR grant QUACO ANR-17-CE40-0007-01.

\section{References}

[ABGM05] T Amand, V Blanchet, B Girard, and X Marie. Coherent control in atoms, molecules and solids. In C Rullière, editor, Femtosecond Laser Pulses, chapter 11. Springer, New York, NY, 2005.

[BCC13] Nabile Boussaïd, Marco Caponigro, and Thomas Chambrion. Weakly coupled systems in quantum control. IEEE Trans. Automat. Control, 58(9):2205-2216, 2013.

[BCC17] N Boussaid, M Caponigro, and T Chambrion. Regular propagators of bilinear quantum systems. Preprint HAL-01016299, 2017.

[BCC19] N Boussaid, M Caponigro, and T Chambrion. On the Ball-Marsden-Slemrod obstruction for bilinear control systems. Preprint hal-01537743, 2019.

[BKMZ94] D. D. Bă̌nov, S. I. Kostadinov, Nguyen Van Minh, and P. P. Zabreı̌ko. Topological equivalence and exponential dichotomy of linear impulsive equations. Internat. J. Theoret. Phys., 33(7):1581-1597, 1994.

[BMS82] J. M. Ball, J. E. Marsden, and M. Slemrod. Controllability for distributed bilinear systems. SIAM J. Control Optim., 20(4):575-597, 1982.

[BR88] Alberto Bressan and Franco Rampazzo. On differential systems with vector-valued impulsive controls. Boll. Un. Mat. Ital. B (7), 2(3):641-656, 1988.

[Bre96] Alberto Bressan. Impulsive control systems. In Boris S. Mordukhovich and Hector J. Sussmann, editors, Nonsmooth Analysis and Geometric Methods in Deterministic Optimal Control, pages 1-22, New York, NY, 1996. Springer New York.

[Bre08] Alberto Bressan. Impulsive control of Lagrangian systems and locomotion in fluids. Discrete Contin. Dyn. Syst., 20(1):1-35, 2008.

[Car11] Rémi Carles. Nonlinear Schrödinger equation with time dependent potential. Commun. Math. Sci., 9(4):937-964, 2011.

[CM05] E Constant and E Mével. Attosecond pulses. In C Rullière, editor, Femtosecond Laser Pulses, chapter 11. Springer, New York, NY, 2005.

[DMR91] Gianni Dal Maso and Franco Rampazzo. On systems of ordinary differential equations with measures as controls. Differential Integral Equations, 4(4):739-765, 1991.

[EG92] Lawrence C. Evans and Ronald F. Gariepy. Measure theory and fine properties of functions. Studies in Advanced Mathematics. CRC Press, Boca Raton, FL, 1992.

[Fuj79] Daisuke Fujiwara. A construction of the fundamental solution for the Schrödinger equation. J. Analyse Math., 35:41-96, 1979. 
[Hel12] E. Helly. Über lineare funktionaloperationen. Wien. Ber., 121:265-297, 1912.

[IL18] P. Indhumathi and A. Leelamani. Existence and uniqueness results for mild solutions of random impulsive abstract neutral partial differential equation over real axis. Appl. Math. J. Chinese Univ. Ser. B, 33(1):71-87, 2018.

[Kat53] T. Kato. Integration of the equation of evolution in a Banach space. J. Math. Soc. Japan, 5:208-234, 1953.

[Mil76] B. M. Miller. Nonlinear impulsive-control problem. Avtomat. i Telemeh., (6):63-72, 1976.

[MR04] Mazyar Mirrahimi and Pierre Rouchon. Controllability of quantum harmonic oscillators. IEEE Trans. Automat. Control, 49(5):745-747, 2004.

[MS10] Paolo Mason and Mario Sigalotti. Generic controllability properties for the bilinear Schrödinger equation. Communications in Partial Differential Equations, 35:685-706, 2010.

[Nat55] I. P. Natanson. Theory of functions of a real variable. Frederick Ungar Publishing Co., New York, 1955. Translated by Leo F. Boron with the collaboration of Edwin Hewitt.

[Sus76] H. J. Sussmann. On generalized inputs and white noise. In 1976 IEEE Conference on Decision and Control including the 15th Symposium on Adaptive Processes, pages 809-814, Dec 1976. 